\title{
Triptans attenuate capsaicin-induced CREB phosphorylation within the trigeminal nucleus caudalis: a mechanism to prevent central sensitization?
}

\author{
Dimos D. Mitsikostas • Yolande E. Knight • \\ Michele Lasalandra $\cdot$ Nikolaos Kavantzas • \\ Peter J. Goadsby
}

Received: 6 February 2011/ Accepted: 9 May 2011/Published online: 29 May 2011

(c) The Author(s) 2011. This article is published with open access at Springerlink.com

\begin{abstract}
The c-AMP-responsive element binding protein (CREB) and its phosphorylated product (P-CREB) are nuclear proteins expressed after stimulation of pain-producing areas of the spinal cord. There is evidence indicating that central sensitization within dorsal horn neurons is dependent on P-CREB transcriptional regulation. The objectives of the study were to investigate the expression of $\mathrm{P}-\mathrm{CREB}$ in cells in rat trigeminal nucleus caudalis after noxious stimulation and to determine whether pre-treatment with specific anti-migraine agents modulate this expression. CREB and P-CREB labelling was investigated within the trigeminal caudalis by immunohistochemistry after capsaicin stimulation. Subsequently, the effect of i.v. pre-treatment with either sumatriptan $(n=5)$, or naratriptan $(n=7)$ on P-CREB expression was studied. Five animals pre-treated with i.v. normal saline were served as controls. CREB and P-CREB labelling was robust in all animal groups within Sp5C. Both naratriptan and sumatriptan decreased P-CREB expression $(p=0.0003$ and 0.0013) within the Sp5C. Triptans attenuate activation of CREB within the central parts of the trigeminal system,
\end{abstract}

D. D. Mitsikostas · Y. E. Knight · M. Lasalandra .

P. J. Goadsby

Headache Group, Department of Neurology,

University of California, San Francisco,

San Francisco, CA, USA

D. D. Mitsikostas $(\square)$

Neurology Department, Athens Naval Hospital,

77A Vas. Sofias Avenue, 11521 Athens, Greece

e-mail: dmitsikostas@ath.forthnet.gr

N. Kavantzas

1st Department of Pathology, Medical School,

Athens National University, Athens, Greece thereby leading to potential inhibition of central sensitization. P-CREB may serve as a new marker for post-synaptic neuronal activation within Sp5C in animal models relevant to migraine.

Keywords CREB - Sumatriptan · Naratriptan · Capsaicin $\cdot$ Migraine $\cdot$ Sensitization

\section{Introduction}

The c-AMP-responsive element binding protein (CREB) is a transcription factor that regulates the expression of genes important for adaptive neuronal responses [1] as well as complex functions such as learning and memory [2]. Upon extracellular stimuli multiple kinases, such as protein kinase $\mathrm{A}$, protein kinase $\mathrm{C}$ and casein kinase II, phosphorylate CREB at serine 133 (P-CREB) leading to activation of immediate early gene $c$-fos $[3,4]$. Fos protein expression can then be used as a marker of neuronal activation within brainstem and spinal nociceptive pathways [5]. CREB also target other genes, such as brain-derived neurotrophic factor (BDNF), that is significantly involved in depression, serving as potential marker for treatment together with P-CREB [6].

Like $c$-fos, CREB signalling is also involved in pain processing, e.g. at the level of spinal cord in a study of tissue injury-induced inflammation and hyperalgesia [7]. Likewise, at the brainstem level, in vitro evidence suggests that P-CREB within the rat trigeminal ganglion is regulated by calcitonin gene-related peptide (CGRP) induced by activation of either the adenosine $\mathrm{A}_{1}$ receptor or the $\mathrm{P} 2 \mathrm{X} 3$ receptor $[8,9]$. In addition, it has been proposed that inhibition of CREB prior to its nuclear translocation may prevent the slowly developing onset of sensitization within 
the brainstem [9], as other studies indicate that central sensitization within the dorsal root neurons is mediated via P-CREB-mediated transcriptional regulation [10].

Allodynia has been recognized in migraine since the nineteenth century [11], with clinic- [12] and population[13] based studies showing that it is seen in about twothirds of patients. Allodynia is a clinical reflection of sensitization, and both central and peripheral sensitization are important insofar as they both influence attacks and perhaps disease progression $[14,15]$. Sensitization of peripheral trigeminovascular neurons that innervate the meninges may be crucial for the development of throbbing in the initial phase of migraine following by sensitization of central trigeminal neurons within the trigeminal nucleus caudalis [16]. In previous work, sumatriptan did not change P-CREB induced by forskolin in cultured neurons taken from adult rat trigeminal ganglions, nor inhibit CGRP release at the same model, as may expected [8], although the role of CGRP on sensitization is unclear [17]. An interesting question remains as to whether triptans modulate phosphorylation of CREB within neurons of the trigeminal nucleus caudalis in vivo. We, therefore, mapped $\mathrm{P}$-CREB expression within rat trigeminal nucleus caudalis after capsaicin stimulation of the trigeminovascular system, and determined the effect of triptans, serotonin $5-\mathrm{HT}_{1 \mathrm{~B} / 1 \mathrm{D}}$ receptor agonists. We show capsaicin stimulation of the trigeminal system activates P-CREB within trigeminal nucleus caudalis and both sumatriptan and naratriptan inhibit induced P-CREB at doses relevant to clinical practice.

\section{Methods}

Male Sprague-Dawley rats (240-300 g) were housed under diurnal lighting and allowed food and water ad libitum. All experiments were conducted consistent with the UK Home Office Animals (Scientific Procedures) Act (1986).

\section{CREB and P-CREB mapping}

with immunohistochemistry

Eight rats were anaesthetized with pentobarbital i.p. $(60 \mathrm{mg} / \mathrm{kg})$ and the femoral vein was cannulated for physiology and drug treatments. Ten minutes later two rats were killed (intact animals), whereas a craniotomy was performed in the remaining six animals. After craniotomy $0.1,1$ or $10 \mu \mathrm{M}$ capsaicin in a cotton ball was directly applied onto the right middle meningeal artery (MMA) for $5 \min (n=2$ for each capsaicin dose). Ten minutes after the end of stimulation period, the animals were killed by pentobarbital $(100 \mathrm{mg} / \mathrm{kg}$, i.v.) and perfused with PBS followed by paraformaldehyde. Brainstems were removed and kept in $30 \%$ sucrose until sectioning in a freezing microtome $(5 \mu \mathrm{m})$ followed by immunohistochemistry (avidin-biotin procedure).

Several dilutions of P-CREB and CREB antibodies (Cell Signalling Technology, cat\# 9191 and 9192, respectively) were tested to establish 1:100 as optimal. Biotinylated sheep anti-rabbit serum (vector) served as a secondary antibody (1:600). Tissues from two non-stimulated animals were used as controls. The brainstems from two other intact animals, where only anaesthesia followed by killing was performed after $30 \mathrm{~min}$, were processed for immunohistochemistry to study the effect of surgery on CREB and P-CREB expression at a comparable timing. Two additional animals were treated as above following exactly the same procedure but killed $2 \mathrm{~h}$ after capsaicin stimulation $(1 \mu \mathrm{M})$. Their brainstems were prepared for fos protein immunohistochemistry at the level of the trigeminal nucleus caudalis [18] to determine if the stimulation was sufficient to activate the trigeminal neurons. Although we refer to the labelling as CREB and P-CREB we cannot exclude cross-reactivity of the antibody so that CREB-like and P-CREB-like may be better terms.

\section{Pharmacological studies}

The effect of i.v. pre-treatment with either sumatriptan $1 \mathrm{mg} \mathrm{kg}^{-1}(n=5)$, or naratriptan $1 \mathrm{mg} \mathrm{kg}^{-1}(n=7)$ on P-CREB expression within Sp5C was studied following the above described procedure. Five animals pre-treated with i.v. normal saline given at the same volume as the drugs served as controls.

\section{Physiology}

Physiological monitoring was carried out in all animals, including arterial pressure (MABP), heart rate (HR) and rectal temperature.

CREB and P-CREB immunoreactive cell counting (image analysis method)

Images were acquired using a Zeiss Axiolab microscope (Carl Zeiss GmbH Jena, Germany) with a mechanical stage, fitted with a Sony-iris CCD videocamera (Sony Corp. Tokyo, Japan). The video camera was connected to a PC (Pentium IV-based) loaded with appropriate image analysis software (Sigma Scan Pro 5.0, Science, Germany). Slides were examined at a magnification $\times 40$. More specifically, in each case the cellular area of the trigeminal nucleus caudalis was selected according to the atlas of Paxinos and Watson [19] and then the number of P-CREB immunostaining cells were calculated via a semiautomatic 
procedure using the above-mentioned image analysis software. Prototypes of positively stained neurons were carefully selected by an expert (DDM) and then labelled cells were counted within the pre-defined areas by the software automatically. Counting was performed by an investigator blind to drug treatment (NK). Twelve coronal sections of the trigeminal nucleus caudalis were selected for counting from each animal; four consecutive slides at the obex level, four at level $-2 \mathrm{~mm}$ and other four at the level $-6 \mathrm{~mm}(\mathrm{C} 2)$ [20]. The mean number was calculated and used for statistics for each animal.

\section{Results}

Blood pressure and heart rate (not shown) did not differ between animals, except that both the animals treated with high capsaicin dose $(10 \mu \mathrm{M})$ died.

\section{CREB and P-CREB mapping}

with immunohistochemistry

In the intact animals CREB but not P-CREB induction was detected bilaterally within all parts of trigeminal nucleus caudalis (data not shown). After craniotomy, CREB was detected bilaterally within almost all the brainstem nuclei including the trigeminal nucleus caudalis, solitary tract, area postrema, lateral reticular nucleus, inferior olive nucleus, within all the laminae. P-CREB was not detected within the trigeminal nucleus caudalis. At the dose of $1 \mu \mathrm{M}$ of capsaicin CREB labelling increased in all the above-mentioned nuclei and P-CREB labelling was found as well within the ipsilateral side of trigeminal nucleus caudalis laminae I and II (5-30 cells per section within the obex and the rostal parts down to $\mathrm{C}_{2}$ level). Neurons in lamina $\mathrm{X}$ were labelled as well, whereas only a few contralateral neurons were labelled at laminae I, II. Capsaicin $0.1 \mu \mathrm{M}$ induced weak P-CREB labelling in cells within laminae I and II (1-5 neurons per section) (Fig. 1). Fos protein-labelled neurons were observed within Sp5C laminae I and II $2 \mathrm{~h}$ after $1 \mu \mathrm{mol}$ capsaicin stimulation. Fos labelling was robust and clear. After capsaicin stimulation $(0.1$ and $1 \mu \mathrm{M})$ one animal died in each dose (mortality rate 10\%). Therefore, the dose of $1 \mu \mathrm{M}$ of capsaicin was selected for consecutive drug testing.

\section{Pharmacological studies}

Pre-treatment with i.v. sumatriptan and naratriptan at a dose of $1 \mathrm{mg} \mathrm{kg}^{-1}$ significantly decreased capsaicininduced P-CREB expression within laminae I and II of trigeminal nucleus caudalis compared to vehicle pre-treated animals (Figs. 2, 3). Two treated animals died (mortality rate $10.5 \%$ ). The physiological data were not different between three groups (data not shown).
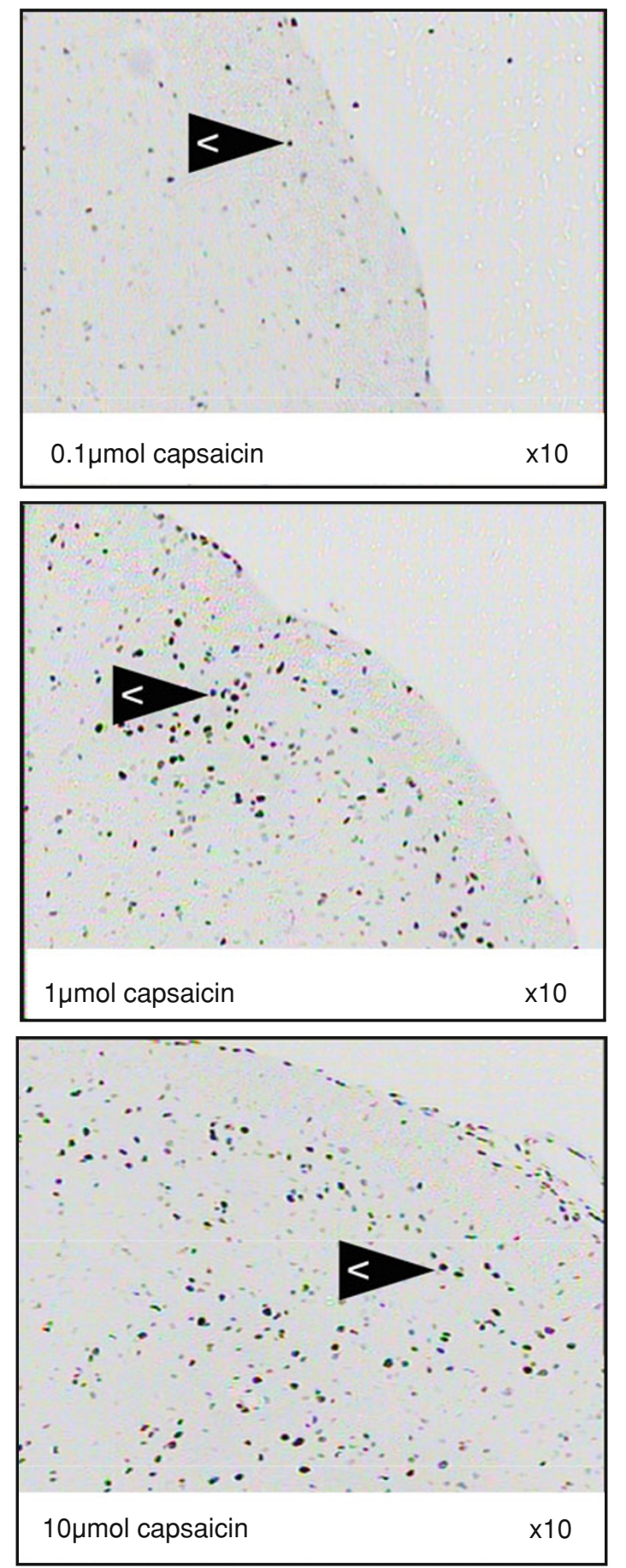

Fig. 1 Micro-photographs show P-CREB expression within the rat trigeminal nucleus caudalis (Sp5C) after capsaicin stimulation (obex). Capsaicin applied onto the middle meningeal artery (MMA) for $5 \mathrm{~min}$ at three different doses $(1,0.1$ and $10 \mu \mathrm{M}, n=2$ for each dose). P-CREB-labelled neurons are shown (arrows) within the rat trigeminal nucleus caudalis (obex) $10 \mathrm{~min}$ after the end of stimulation. Avidin-biotin immunohistochemistry 


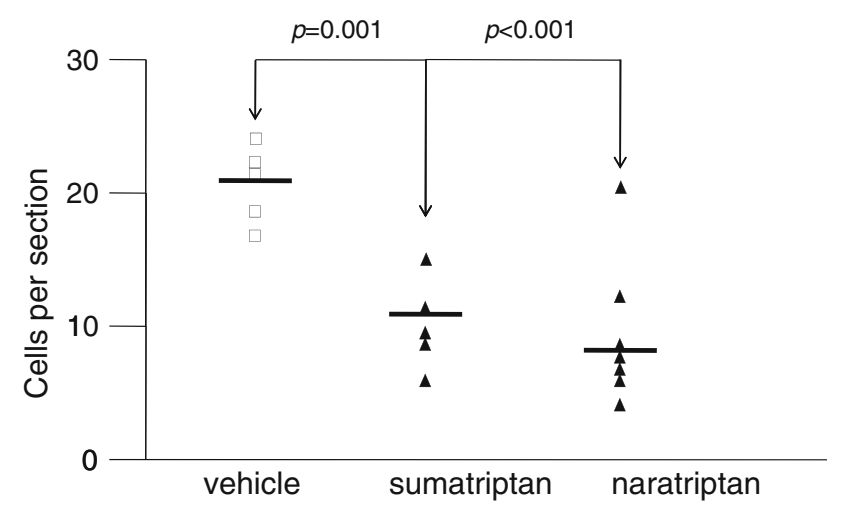

Fig. 2 Treatment with both sumatriptan and naratriptan attenuates CREB activation within trigeminal nucleus caudalis. Sumatriptan $(n=5)$ and naratriptan $(n=7)$ at a dose of $1 \mathrm{mg} \mathrm{kg}^{-1}$ i.v. significantly decreased capsaicin $(1 \mu \mathrm{mol})$ induced P-CREB expression within trigeminal nucleus caudalis compared to vehicle treated animals $(n=5)$

\section{Discussion}

We show here that stimulation of the peripheral parts of the rat trigeminovascular system, first-order trigeminal neurons, by capsaicin-induced CREB phosphorylation within the second-order neurons at the brainstem in the trigeminal nucleus caudalis. To our knowledge, this is the first in vivo study mapping the CREB and P-CREB expression within the trigeminal nucleus caudalis after noxious stimulus. Furthermore, the anti-migraine drugs sumatriptan and naratriptan prevented brainstem CREB activation.

\section{Methodological limitations}

Preabsorption controls for anti-CREB and anti-P-CREB were not performed, to prove their specificity to the protein using the exact immunohistochemical protocol except for the addition of the immunizing peptide. However, we followed the manufacturer's immunohistochemical protocols and the P-CREB labelling distribution within the painproducing areas of the trigeminal system supports the specificity of the antibodies used. Several time points for CREB and P-CREB expression were not tested to see if the 10-min window was optimal for drug testing. We followed suggestions based on previous experiments [7,21] showing that this time window, 10 min after stimulation of MMA to observe phosphorylation of CREB within trigeminal nucleus caudalis, was appropriate. The mortality rate was slightly high (10\%) but acceptable. Finally, a TRPV1 antagonist could be used to show a reverse effect. We tested only one dose of triptans, not several to show a possible dose-response related effect. Also the effect of triptans to inhibit other stimuli (mechanical distension or thermal stimuli or other chemical stimuli) was not studied.

CREB and P-CREB mapping within the trigeminal system

Capsaicin $1 \mu \mathrm{mol}$ applied on the MMA-induced P-CREB immunohistochemistry within the ipsilateral brain stem trigeminal nucleus caudalis, as it has been shown with $c$-fos [5]. P-CREB within Sp5C was relatively pain-specific because of the particular distribution within the laminae I and II, that was clear for counting and with good signal to noise for drug testing. Physiological monitoring showed that the microsurgical procedure and the capsaicin stimulation were tolerated well by the pentobarbital anaesthetized rats. Previous in vitro experiments showed P-CREB expression within the rat trigeminal ganglion neurons induced by forskolin [8]. Recently, activation of CREB

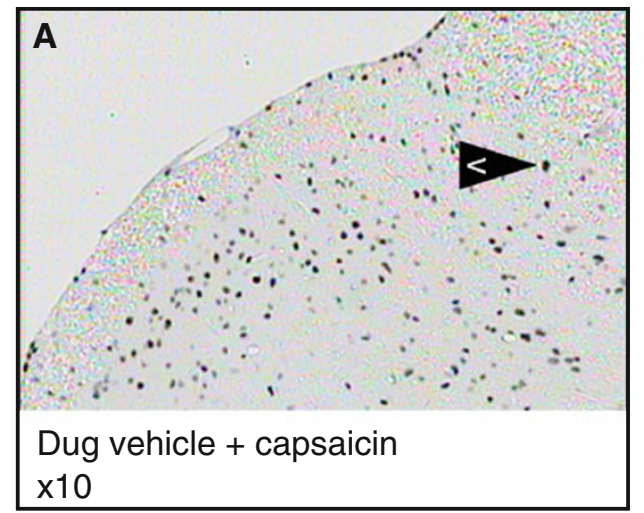

Fig. 3 Micro-photographs show P-CREB expression within the rat trigeminal nucleus caudalis (Sp5C) after capsaicin stimulation and sumatriptan treatment (obex). Normal saline $(n=5)$ or sumatriptan $1 \mathrm{mg} \mathrm{kg}^{-1}(n=5)$ was i.v. administered in rats and 10 min later $1 \mu \mathrm{M}$ capsaicin applied onto the middle meningeal artery (stimulation lasted for 5 mins). Ten minutes after the end of stimulation the

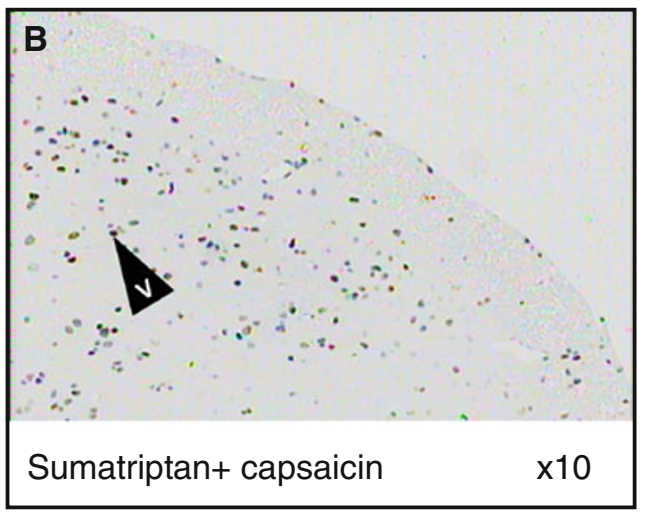

animals were killed and preceded for immunohistochemistry (avidinbiotin procedure). Panel $A$ sample from a vehicle + capsaicin-treated animal. Panel $B$ sample from a sumatriptan + capsaicin-treated $\left(1 \mathrm{mg} \mathrm{kg}^{-1}\right)$ animal. P-CREB labelled neurons are shown (arrows) within the rat trigeminal nucleus caudalis (obex) 
within mouse cultured trigeminal ganglion neurons was seen after treatment with CGRP via $\mathrm{Ca}^{2+}$-dependent mechanisms [9]. We had the opportunity to look for CREB and P-CREB expression within the ipsilateral trigeminal ganglia after capsaicin stimulation; we did not observe P-CREB. Apparently the CREB cascade exists in rat trigeminal ganglion cells, although how to induce it in vivo still remains unclear and needs further investigation.

Triptan effects on P-CREB within trigeminal nucleus caudalis

Both sumatriptan and naratriptan prevented capsaicininduced P-CREB within the trigeminal nucleus caudalis as previously shown with capsaicin-induced $c$-fos [22, 23] indicating that the P-CREB approach yields similar data. At the first neuron level, however, in vitro studies failed to show an effect of sumatriptan on forskolin-induced CREB activation [8], suggesting that sumatriptan triggers the suppressive transcriptional cascade only within the central parts of the trigeminal system, not in the periphery. No such model for $c$-fos exists to compare to, since $c$-fos is not expressed within trigeminal ganglion cells [5]. To better explore the effect of triptans on P-CREB, two drugs with somewhat different pharmacological properties have been tested: the hydrophilic sumatriptan, which does not penetrate the blood brain barrier in normal circumstances, and the tenfold more lipophilic naratriptan [24]. Because both drugs share almost similar affinity to 5-HT1 receptors, we used the same doses for both drugs. The clinically relevant doses are different although this may be simply a matter of absorption. Both triptans attenuated $\mathrm{P}$-CREB regardless of their differences. It is important, however, to understand the molecular mechanism of the action of triptans on neurons. Triptans not only increase intracellular $\mathrm{Ca}^{2+}$ leading in inhibition of CGRP gene transcription [25, 26], but also down regulate gene transcription generally within trigeminal nucleus caudalis cells by blocking CREB phosphorylation and $c$-fos expression, resulting in depression of brainstem nociceptive neurons, as has been shown using electrophysiological methods [27, 28]. These findings may partially explain the effect of triptans on the central sensitization process seen in migraine.

\section{Central sensitization and P-CREB}

It has been speculated that triptans can successfully abort migraine as long as central sensitization still depends on incoming signals from the periphery, but not after central sensitization becomes self-sufficient [29]. Since this hypothesis still lacks good documentation, it would be interesting to test the effect of triptans in vivo within the trigeminal ganglion neurons. However, only electrophysiological studies can be performed, and these techniques are limited to short lasting neuronal alterations. One of the aims of this study was to explore an in vivo approach to investigate the longer time course effects of triptans within both trigeminal ganglion and trigeminal nucleus caudalis neurons, such as effects on gene transcription. Unfortunately trigeminal ganglion cells did not show sufficient labelling to explore this question further. Yet, the findings of our study may serve as vehicle to better understand the effect of triptans on slowly developing central sensitization within trigeminal nucleus caudalis.

Gene transcription is crucial for long lasting neuronal changes triggered by several extracellular signals. In the case of CREB, multiple molecules or proteins, such as CGRP [9], lead to its phosphorylation, via activation of several kinases [1, 30-32]. After phosphorylation CREB activates other immediate early genes like $c$-fos, molecules important for synaptic function like brain-derived neurotrophic factor and neuronal nitric oxide [33], which are all involved in pain transmission. Thus, selective blocking of CREB activation before its nuclear translocation would be important in inhibiting pain signalling acutely and also in blocking the sensitization cascade triggered by P-CREB, therefore reducing escalation of pain signalling [9]. In other words this would be pivotal for reducing the escalation/ persistence of pain due to central sensitization, such as in migraine. In addition, P-CREB may also serve as marker of neuronal presynaptic activation within the trigeminovascular system in animal models of migraine biology, like fos protein.

C-fos and P-CREB as markers for trigeminal caudalis activation in animal models of migraine biology

$C$-fos encodes a nuclear protein that regulates the transcription of other target genes and of its one promoter. Its detection within the brainstem and spinal cord neurons is the best studied technique to map the postsynaptic nociceptive pathways in numerous cephalic pain models $[5,18$, 34]. In the field of migraine research fos protein immunoreactivity offers a method to identify subpopulations of neurons activated in response to noxious stimuli and identify related nociceptive pathways [5]. The majority of studies have employed this technique to map neuronal activation not only throughout the trigeminovascular system [35-39], but also higher structures involved in the ascending and descending modulatory control of pain [6, 40-42], thus, greatly enhancing our understanding of the pathophysiology of migraine. As with other models of the components of migraine the use of fos expression has certain limitations [43]. The major one being the model can only be as good as the stimulus since it is the stimulus that 
drives the expression of fos protein. Several intracranial structures have been stimulated chemically or electrically to induce fos within trigeminal nucleus caudalis, including the meninges [18, 20, 22, 23], trigeminal ganglion [44], the superior sagittal sinus [35] and middle meningeal artery [45]. It seems that the activation of specific structures of the trigeminovascular system (e.g. the superior sagittal sinus) leads to more clinically relevant conclusions [35, 46]. It is noteworthy that induction of fos to quantifiable levels requires a strong consistent stimulation that is often not physiological [47, 48]. It must also be remembered that c-fos is not expressed in all neurons as with the dorsal root ganglion cells [34], thus, lack of fos protein expression does not equate to lack of neuronal activity. Technical difficulties in inducing $c$-fos expression within trigeminal nucleus caudalis are also important [49]. One final limitation of the model is seen via direct activation of the antinociceptive descending pathways, which elicits $c$-fos expression in spinal neurons even in the absence of any nociceptive input [50].

P-CREB on the other hand displays several advantages over $c$-fos as a neuronal marker of post-synaptic activation within trigeminal nucleus caudalis. So far, activation of CREB within the trigeminal nucleus caudalis, or trigeminal ganglion in vitro, has been seen only after specific activation of nociceptive neurons, thus P-CREB within laminae I and II seems to be a direct effect of the noxious stimulus used. In addition, the time window needed for phosphorylation of CREB is much shorter, up to $10 \mathrm{~min}$, compared to 120 min needed for $c$-fos expression, leading potentially to lower animal mortality rates. Although only stimulation of middle meningeal artery has been used, theoretically all the other parts of the peripheral trigeminal system can be used to induce P-CREB. The fact that P-CREB expression has been detected within trigeminal ganglion, although only in vitro, raises new possibilities for pharmaocological studies within both the peripheral and central parts of the trigeminal system. Further investigation is required though to induce and map P-CREB in trigeminal ganglion cells in vivo.

\section{Conclusions}

P-CREB is a transcriptional factor involved in sensitization of nociceptive cells. Noxious stimulation of peripheral parts of the trigeminal system induces P-CREB within trigeminal nucleus caudalis. Triptans inhibit this activation. Thus, triptans might prevent central sensitization by attenuating P-CREB-mediated transcriptional pathways. In addition, P-CREB may serve as a new marker for postsynaptic neuronal activation within trigeminal nucleus caudalis in animal models of migraine biology.
Acknowledgments This work was partly supported by an educational grant from Hellenic Navy, General Staff (DDM).

\section{Conflict of interest None.}

Open Access This article is distributed under the terms of the Creative Commons Attribution License which permits any use, distribution and reproduction in any medium, provided the original author(s) and source are credited.

\section{References}

1. Ginty DD, Bonni A, Greenberg ME (1994) Nerve growth factor activates a Ras-dependent protein kinase that stimulates c-fos transcription via phosphorylation of CREB. Cell 77:713-725

2. Frank DA, Greenberg ME (1994) CREB: a mediator of long-term memory from mollusks to mammals. Cell 79:5-8

3. Gonzalez GA, Yamamoto KK, Fischer WH, Karr D, Menzel P, Biggs W 3rd, Vale WW, Montminy MR (1989) A cluster of phosphorylation sites on the cyclic AMP-regulated nuclear factor CREB predicted by its sequence. Nature 337:749-752

4. Yamamoto KK, Gonzalez GA, Biggs WH 3rd, Montminy MR (1988) Phosphorylation-induced binding and transcriptional efficacy of nuclear factor CREB. Nature 334:494-498

5. Mitsikostas DD, Sanchez del Rio M (2001) Receptor systems mediating c-fos expression within trigeminal nucleus caudalis in animal models of migraine. Brain Res Brain Res Rev 35:20-35

6. Koch JM, Hinze-Selch D, Stingele K, Huchzermeier C, Göder R, Seeck-Hirschner M, Aldenhoff JB (2009) Changes in CREB phosphorylation and BDNF plasma levels during psychotherapy of depression. Psychother Psychosom 78:187-192

7. Ji RR, Rupp F (1997) Phosphorylation of transcription factor CREB in rat spinal cord after formalin-induced hyperalgesia: relationship to c-fos induction. J Neurosci 17:1776-1785

8. Carruthers AM, Sellers LA, Jenkins DW, Jarvie EM, Feniuk W, Humphrey PP (2001) Adenosine A(1) receptor-mediated inhibition of protein kinase A-induced calcitonin gene-related peptide release from rat trigeminal neurons. Mol Pharmacol 59:15331541

9. Simonetti M, Giniatullin R, Fabbretti E (2008) Mechanisms mediating the enhanced gene transcription of $\mathrm{P} 2 \mathrm{X} 3$ receptor by calcitonin gene-related peptide in trigeminal sensory neurons. J Biol Chem 283:18743-18752

10. Kawasaki Y, Kohno T, Zhuang ZY, Brenner GJ, Wang H, Van Der Meer C, Befort K, Woolf CJ, Ji RR (2004) Ionotropic and metabotropic receptors, protein kinase $\mathrm{A}$, protein kinase $\mathrm{C}$, and Src contribute to $\mathrm{C}$-fiber-induced ERK activation and cAMP response element-binding protein phosphorylation in dorsal horn neurons, leading to central sensitization. J Neurosci 24:8310 8321

11. Gowers WR (1888) A manual of diseases of the nervous system. P. Blakiston, Son \& Co, Philadelphia

12. Selby G, Lance JW (1960) Observations on 500 cases of migraine and allied vascular headache. J Neurol Neurosurg Psychiatry 23:23-32

13. Bigal ME, Ashina S, Burstein R, Reed ML, Buse D, Serrano D, Lipton RB, AMPP Group (2008) Prevalence and characteristics of allodynia in headache sufferers: a population study. Neurology 70:1525-1533

14. Burstein R (2001) Deconstructing migraine headache into peripheral and central sensitization. Pain 89:107-110

15. Cooke L, Eliasziw M, Becker WJ (2007) Cutaneous allodynia in transformed migraine patients. Headache 47:531-539 
16. Burstein R, Levy D, Jakubowski M (2005) Effects of sensitization of trigeminovascular neurons to triptan therapy during migraine. Rev Neurol (Paris) 161:658-660

17. Levy D, Burstein R, Strassman AM (2005) Calcitonin generelated peptide does not excite or sensitize meningeal nociceptors: implications for the pathophysiology of migraine. Ann Neurol 58:698-705

18. Mitsikostas DD, Sanchez del Rio M, Moskowitz MA, Waeber C (1999) Both 5-HT1B and 5-HT1F receptors modulate c-fos expression within rat trigeminal nucleus caudalis. Eur J Pharmacol 369:271-277

19. Paxinos G, Watson C (1997) The rat brain in stereotaxic coordinates. Academic Press, San Diego

20. Mitsikostas DD, Sanchez del Rio M, Waeber C, Moskowitz MA, Cutrer FM (1998) The NMDA receptor antagonist MK-801 reduces capsaicin-induced c-fos expression within rat trigeminal nucleus caudalis. Pain 76:239-248

21. Ji RR, Bose CM, Lesuisse C et al (1998) Specific agrin isoforms induce cAMP response element binding protein phosphorylation in hippocampal neurons. J Neurosci 18:9695-9702

22. Mitsikostas DD, Sanchez del Rio M, Waeber C (2002) 5-Hydroxytryptamine(1B/1D) and 5-hydroxytryptamine1F receptors inhibit capsaicin-induced c-fos immunoreactivity within mouse trigeminal nucleus caudalis. Cephalalgia 22:384-394

23. Mitsikostas DD, Sanchez del Rio M, Waeber C, Huang Z, Cutrer FM, Moskowitz MA (1999) Non-NMDA glutamate receptors modulate capsaicin induced c-fos expression within trigeminal nucleus caudalis. Br J Pharmacol 127:623-630

24. Tfelt-Hansen P, De Vries P, Saxena PR (2000) Triptans in migraine: a comparative review of pharmacology, pharmacokinetics and efficacy. Drugs 60:1259-1287

25. Durham PL, Russo AF (1999) Regulation of calcitonin generelated peptide secretion by a serotonergic antimigraine drug. J Neurosci 19:3423-3429

26. Durham PL, Sharma RV, Russo AF (1997) Repression of the calcitonin gene-related peptide promoter by 5 -HT1 receptor activation. J Neurosci 17:9545-9553

27. Goadsby PJ, Akerman S, Storer RJ (2001) Evidence for postjunctional serotonin (5-HT1) receptors in the trigeminocervical complex. Ann Neurol 50:804-807

28. Storer RJ, Goadsby PJ (1997) Microiontophoretic application of serotonin (5HT)1B/1D agonists inhibits trigeminal cell firing in the cat. Brain 120:2171-2177

29. Burstein R, Jakubowski M (2004) Analgesic triptan action in an animal model of intracranial pain: a race against the development of central sensitization. Ann Neurol 55:27-36

30. Montminy M (1997) Transcriptional regulation by cyclic AMP. Annu Rev Biochem 66:807-822

31. Sheng M, Thompson MA, Greenberg ME (1991) CREB: a $\mathrm{Ca}(2+)$-regulated transcription factor phosphorylated by calmodulin-dependent kinases. Science 252:1427-1430

32. Xing J, Ginty DD, Greenberg ME (1996) Coupling of the RASMAPK pathway to gene activation by RSK2, a growth factorregulated CREB kinase. Science 273:959-963

33. Lonze BE, Ginty DD (2002) Function and regulation of CREB family transcription factors in the nervous system. Neuron 35:605-623

34. Hunt SP, Pini A, Evan G (1987) Induction of c-fos-like protein in spinal cord neurons following sensory stimulation. Nature 328:632-634
35. Goadsby PJ, Hoskin KL (1997) The distribution of trigeminovascular afferents in the nonhuman primate brain Macaca nemestrina: a c-fos immunocytochemical study. J Anat 190:367375

36. Hoskin KL, Bulmer DC, Goadsby PJ (1999) Fos expression in the trigeminocervical complex of the cat after stimulation of the superior sagittal sinus is reduced by L-NAME. Neurosci Lett 266:173-176

37. Kaube H, Keay KA, Hoskin KL, Bandler R, Goadsby PJ (1993) Expression of c-Fos-like immunoreactivity in the caudal medulla and upper cervical spinal cord following stimulation of the superior sagittal sinus in the cat. Brain Res 629:95-102

38. Strassman AM, Mineta Y, Vos BP (1994) Distribution of fos-like immunoreactivity in the medullary and upper cervical dorsal horn produced by stimulation of dural blood vessels in the rat. J Neurosci 14:3725-3735

39. Sugimoto T, He YF, Xiao C, Ichikawa H (1998) c-fos induction in the subnucleus oralis following trigeminal nerve stimulation. Brain Res 783:158-162

40. Benjamin L, Levy MJ, Lasalandra MP, Knight YE, Akerman S, Classey JD, Goadsby PJ (2004) Hypothalamic activation after stimulation of the superior sagittal sinus in the cat: a Fos study. Neurobiol Dis 16:500-505

41. Hoskin KL, Bulmer DC, Lasalandra M, Jonkman A, Goadsby PJ (2001) Fos expression in the midbrain periaqueductal grey after trigeminovascular stimulation. J Anat 198:29-35

42. Malick A, Jakubowski M, Elmquist JK, Saper CB, Burstein R (2001) A neurohistochemical blueprint for pain-induced loss of appetite. Proc Natl Acad Sci USA 98:9930-9935

43. Bergerot A, Holland PR, Akerman S, Bartsch T, Ahn AH, MaassenVanDenBrink A, Reuter U, Tassorelli C, Schoenen J, Mitsikostas DD, van den Maagdenberg AM, Goadsby PJ (2006) Animal models of migraine: looking at the component parts of a complex disorder. Eur J Neurosci 24:1517-1534

44. Clayton JS, Gaskin PJ, Beattie DT (1997) Attenuation of Fos-like immunoreactivity in the trigeminal nucleus caudalis following trigeminovascular activation in the anaesthetised guinea-pig. Brain Res 775:74-80

45. Hoskin KL, Zagami AS, Goadsby PJ (1999) Stimulation of the middle meningeal artery leads to Fos expression in the trigeminocervical nucleus: a comparative study of monkey and cat. J Anat 194:579-588

46. May A, Goadsby PJ (2001) Substance P receptor antagonists in the therapy of migraine. Expert Opin Investig Drugs 10:673-678

47. Bullitt E, Lee CL, Light AR, Willcockson H (1992) The effect of stimulus duration on noxious-stimulus induced c-fos expression in the rodent spinal cord. Brain Res 580:172-179

48. Lima D, Avelino A (1994) Spinal c-fos expression is differentially induced by brief or persistent noxious stimulation. Neuroreport 5:1853-1856

49. Hoskin KL, Goadsby PJ (1999) Exposure and isolation of the superior sagittal sinus elicits Fos in the trigeminal nucleus caudalis and dorsal horn of the cervical spinal cord: how long should you wait? Brain Res 824:133-135

50. Bett K, Sandkuhler J (1995) Map of spinal neurons activated by chemical stimulation in the nucleus raphe magnus of the unanesthetized rat. Neuroscience 67:497-504 